\title{
ASYMPTOTIC SOLUTIONS OF A PARABOLIC EQUATION NEAR SINGULAR POINTS OF $A$ AND $B$ TYPES
}

\author{
Sergey V. Zakharov \\ Krasovskii Institute of Mathematics and Mechanics, \\ Ural Branch of the Russian Academy of Sciences, \\ 16 S. Kovalevskaya str., Ekaterinburg, Russia, 620990 \\ svz@imm.uran.ru
}

\begin{abstract}
The Cauchy problem for a quasi-linear parabolic equation with a small parameter multiplying a higher derivative is considered in two cases when the solution of the limit problem has a point of gradient catastrophe. Asymptotic solutions are found by using the Cole-Hopf transform. The integrals determining the asymptotic solutions correspond to the Lagrange singularities of type $A$ and the boundary singularities of type $B$. The behavior of the asymptotic solutions is described in terms of the weighted Sobolev spaces.

Keywords: Quasi-linear parabolic equation, Cole-Hopf transform, Singular points, Asymptotic solutions, Whitney fold singularity, Il'in's universal solution, Weighted Sobolev spaces.
\end{abstract}

\section{Introduction}

Consider the Cauchy problem for a quasi-linear parabolic equation

$$
\begin{gathered}
\frac{\partial u}{\partial t}+\frac{\partial \varphi(u)}{\partial x}=\varepsilon \frac{\partial^{2} u}{\partial x^{2}}, \\
u\left(x, t_{0}\right)=q(x), \quad x \in \mathbb{R},
\end{gathered}
$$

with a small parameter $\varepsilon>0$ in such cases that the solution of the limit problem (for $\varepsilon=0$ ) has a point of gradient catastrophe. The interest of studying the behavior of solutions near singular points is explained, in particular, by the fact that such singular events themselves occupy very small time, however, in many respects determining all subsequent behavior of the system. The asymptotic behavior of solutions in neighborhoods of singular points is directly connected with constructing an approximation in neighborhoods of shock waves in physical media with a small nonzero viscosity.

Although the types of singular points of solutions are classified in detail [1, ch. 2] and processes of the shock waves formation are studied [2], constructing asymptotic series in the small viscosity parameter $\varepsilon$ for an equation of the general form (0.1) is a separate problem in every specific case. First substantial results for several types of singularities, including the Whitney fold singularity $A_{3}$, were obtained by A. M. Il'in [3, ch. VI], who constructed a complete asymptotics and derived a universal solution near the fold singularity; see formulas (1.1)-(1.2) in the next section.

In the present paper, the statements about asymptotic solutions of the preliminary notice [4] for Lagrange singularities of types $A_{2 n+1}$ and boundary singularities of types $B_{2 n+1}$ with any $n \geqslant 2$ are proved.

\section{Fold singularity}

In Il'in's pioneer paper [5], problem (0.1)-(0.2) was first studied in the case when in the strip $\left\{t_{0} \leqslant t \leqslant T, x \in \mathbb{R}\right\}$ the solution of the limit problem is a function which is smooth everywhere 
except for one smooth line of jump discontinuity $x=s(t), t \geqslant t^{*}$. It is supposed that $\varphi \in C^{\infty}(\mathbb{R})$, $\varphi^{\prime \prime}(u)>0, \varphi(0)=\varphi^{\prime}(0)=0$, and $t_{0}=-1$. For an appropriate choice of the bounded initial function $q \in C^{\infty}(\mathbb{R})$, the singular point $\left(s\left(t^{*}\right), t^{*}\right)$ coincides with the origin and in its neighborhood the stretched variables $\xi=\varepsilon^{-3 / 4} x$ and $\tau=\varepsilon^{-1 / 2} t$ are introduced. An asymptotic expansion of the solution was obtained in the form of the series

$$
u(x, t, \varepsilon)=\sum_{k=1}^{\infty} \varepsilon^{k / 4} \sum_{j=0}^{k-1} w_{k, j}(\xi, \tau) \ln ^{j} \varepsilon^{1 / 4}, \quad \varepsilon \rightarrow+0 .
$$

Observe properties of the leading term of the expansion $\varepsilon^{1 / 4} w_{1,0}(\xi, \tau)$. The coefficient $w_{1,0}(\xi, \tau)$ is found using the Cole-Hopf transform

$$
w_{1,0}(\xi, \tau)=-\frac{2}{\varphi^{\prime \prime}(0) \Lambda(\xi, \tau)} \frac{\partial \Lambda(\xi, \tau)}{\partial \xi}
$$

where

$$
\Lambda(\xi, \tau)=\int_{-\infty}^{+\infty} \exp \left(-\frac{1}{8}\left(z^{4}-2 z^{2} \tau+4 z \xi\right)\right) d z .
$$

The argument of the exponent is a generating family of the Lagrange singularity $A_{3}$, see $[1,6]$.

Theorem 1. The function $w_{1,0}$ satisfies the asymptotic relations

$$
w_{1,0}(\xi, \tau)=\left[\varphi^{\prime \prime}(0)\right]^{-1} H(\xi, \tau)+\sum_{l=1}^{\infty} h_{1-4 l}(\xi, \tau), \quad 3[H(\xi, \tau)]^{2}-\tau \rightarrow \infty,
$$

$(\xi, \tau) \in \Omega_{1}=\mathbb{R}^{2} \backslash\left\{|\xi|<\tau^{\gamma_{1}-1 / 2}, \tau>0,0<\gamma_{1}<2\right\}$, where $H(\xi, \tau)$ is the Whitney fold function, $H^{3}-\tau H+\xi=0, h_{1-4 l}(\xi, \tau)$ are homogeneous functions of power $1-4 l$, relative to $H(\xi, \tau),|\tau|^{1 / 2}$ and $\sqrt{3[H(\xi, \tau)]^{2}-\tau}$, which are polynomials in $H(\xi, \tau), \tau$ and $\left(3[H(\xi, \tau)]^{2}-\tau\right)^{-1}$,

$$
w_{1,0}(\xi, \tau)=\sqrt{\tau}\left(-\frac{\operatorname{th} z}{\varphi^{\prime \prime}(0)}+\sum_{k=1}^{\infty} \tau^{-2 k} q_{k}(z)\right), \quad \tau \rightarrow+\infty,
$$

$(\xi, \tau) \in \Omega_{2}=\left\{|\xi| \tau^{1 / 2}<\tau^{\gamma_{2}}, \gamma_{1}<\gamma_{2}<2\right\}$, where $z=\xi \sqrt{\tau} / 2$, and the coefficients of the series for $k \geqslant 1$ satisfy the estimates $\left|q_{k}(z)\right| \leqslant M_{k}\left(1+|z|^{k}\right)$.

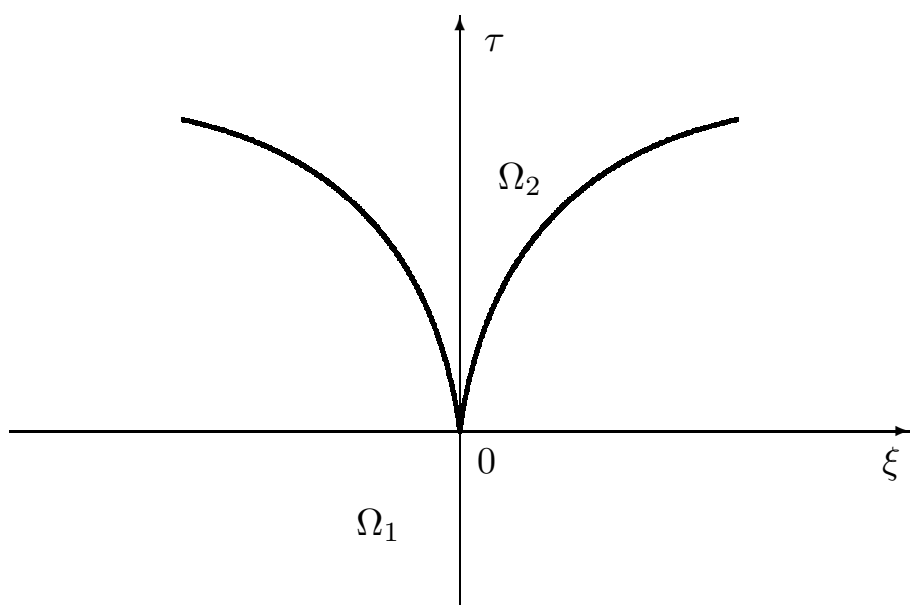

Fig. 1. Domains $\Omega_{1}$ and $\Omega_{2}$.

$\mathrm{P}$ r o o $\mathrm{f}$ of the theorem is based on the calculation of the asymptotics of the integral $\Lambda(\xi, \tau)$ by Laplace's method. In the domain $\Omega_{1}$, see Figure 1 , the essential contribution into the asymptotics 
is given by one local maximum and in the domain $\Omega_{2}$ by two local maxima; see Theorem 4.1 and Lemma 6.1 in $[3$, ch. VI].

Il'in investigated the problem under condition that

$$
\varphi^{\prime}(q(x))=-x+x^{3}+O\left(x^{4}\right), \quad x \rightarrow 0 .
$$

Now consider the following condition

$$
\varphi^{\prime}(q(x))=-x+x^{2 n+1}+O\left(x^{2 n+2}\right), \quad x \rightarrow 0 .
$$

Let us clear out scales of the inner variables, which are introduced using the change

$$
x=\eta \varepsilon^{\sigma}, \quad t=\theta \varepsilon^{\mu}, \quad u(x, t, \varepsilon)=\varepsilon^{\varkappa} u^{*}(\eta, \theta, \varepsilon) .
$$

Since all terms in equation (0.1) should be of the same order, we obtain the relations

$$
-\mu=\varkappa-\sigma=1-2 \sigma
$$

From the characteristic equation $x=y+(t+1) \varphi^{\prime}(q(y))$ with $y \in \mathbb{R}$ being an arbitrary parameter and condition (1.3) we have $x \approx-y t-y^{2 n+1}$, whence by change (1.4) and the relation $u \sim-y$, we obtain $\sigma=\varkappa+\mu=(2 n+1) \varkappa$. From these equalities and relations (1.5) we find

$$
\sigma=\frac{2 n+1}{2 n+2}, \quad \mu=\frac{n}{n+1}, \quad \varkappa=\frac{1}{2 n+2} .
$$

Since $u=O\left(\varepsilon^{1 /(2 n+2)}\right)$, equation (0.1) becomes the Burgers equation, whose solution can be written in the form of the Cole-Hopf transform. Moreover, the coefficient at $s^{2 n+2}$ is determined from the condition of matching the inner asymptotics and the outer expansion

$$
u_{\mathrm{out}} \sim \frac{\varepsilon^{\varkappa} U_{0}(\eta, \theta)}{\varphi^{\prime \prime}(0)}, \quad U_{0}^{2 n+1}-\theta U_{0}+\eta=0 .
$$

To give some description of the behavior of asymptotic solutions, we will use the weighted Sobolev spaces $W_{p, q}^{m}(\mathbb{R})$ with the norm

$$
\|u\|_{W_{p, q}^{m}(\mathbb{R})}=\sum_{l=0}^{m}\left(\int_{-\infty}^{+\infty}(1+|x|)^{-q}\left|\frac{\partial^{l} u(x)}{\partial x^{l}}\right|^{p} d x\right)^{1 / p}
$$

and also the proposed in [7] approach to the definition of an asymptotic solution of an evolutionary differential equation.

Theorem 2. In the domain $\Omega_{\varepsilon}=\left\{(x, t):\left|x \varepsilon^{-\varkappa}\right|+|t|<K \varepsilon^{\mu}, K>0\right\}$ for any natural $n \geqslant 2$ the function

$$
u_{\text {in }}(x, t, \varepsilon)=-2 \varepsilon\left[\varphi^{\prime \prime}(0) V(x, t, \varepsilon)\right]^{-1} \frac{\partial V(x, t, \varepsilon)}{\partial x},
$$

where

$$
\begin{gathered}
V(x, t, \varepsilon)=\int_{-\infty}^{+\infty} \exp \left(-\frac{2^{2 n} s^{2 n+2}}{n+1}+\frac{t s^{2}}{\varepsilon^{\mu}}-\frac{x s}{\varepsilon^{\sigma}}\right) d s \\
\sigma=\frac{2 n+1}{2 n+2}, \quad \mu=\frac{n}{n+1}, \quad \varkappa=\frac{1}{2 n+2}
\end{gathered}
$$


is an asymptotic solution of equation (0.1) in the following sense:

$$
\frac{\partial u_{\text {in }} / \partial t+\partial \varphi\left(u_{\text {in }}\right) / \partial x-\varepsilon \partial^{2} u_{\text {in }} / \partial x^{2}}{\sup _{(x, t) \in \Omega_{\varepsilon}}\left\{\left|\partial u_{\text {in }} / \partial t\right|+\left|c \partial \varphi\left(u_{\text {in }}\right) / \partial x\right|+\left|\varepsilon \partial^{2} u_{\text {in }} / \partial x^{2}\right|\right\}}=O\left(\varepsilon^{\varkappa}\right), \quad \varepsilon \rightarrow+0 .
$$

For $(m+1) p<(2 n+1)(q-1)$ and any fixed $t, u_{\text {in }}(\cdot, t, \varepsilon) \in W_{p, q}^{m}(\mathbb{R})$ and $\left\|u_{\text {in }}(\cdot, t, \varepsilon)\right\|_{W_{p, q}^{m}(\mathbb{R})} \sim \varepsilon^{-m}$ as $\varepsilon \rightarrow+0$.

P r o o f. Formula (1.9) implies that the function $V(x, t, \varepsilon)$ satisfies the heat equation

$$
\frac{\partial V}{\partial t}=\varepsilon \frac{\partial^{2} V}{\partial x^{2}}
$$

it follows that function (1.8) is the exact solution of the Burgers equation:

$$
\frac{\partial u_{\text {in }}}{\partial t}+\varphi^{\prime \prime}(0) u_{\text {in }} \frac{\partial u_{\text {in }}}{\partial x}=\varepsilon \frac{\partial^{2} u_{\text {in }}}{\partial x^{2}}
$$

since $u_{\text {in }}$ has the form of the Cole-Hopf transform (it can also be established by direct substitition). Then using Taylor's formula $\varphi^{\prime}(u)=\varphi^{\prime \prime}(0) u+O\left(u^{2}\right), u \rightarrow 0$, we easily obtain the following estimate:

$$
\frac{\partial u_{\text {in }}}{\partial t}+\frac{\partial \varphi\left(u_{\text {in }}\right)}{\partial x}-\varepsilon \frac{\partial^{2} u_{\text {in }}}{\partial x^{2}}=\varphi^{\prime}\left(u_{\text {in }}\right) \frac{\partial u_{\text {in }}}{\partial x}-\varphi^{\prime \prime}(0) u_{\text {in }} \frac{\partial u_{\text {in }}}{\partial x}=O\left(u_{\text {in }}^{2} \frac{\partial u_{\text {in }}}{\partial x}\right) .
$$

It is also elementary checked that

$$
u_{\mathrm{in}}^{2}=\frac{4 \varepsilon^{2-2 \sigma}}{\left[\varphi^{\prime \prime}(0)\right]^{2}} \frac{\left(V_{\eta}\right)^{2}}{V^{2}}, \quad \frac{\partial u_{\mathrm{in}}}{\partial x}=-\frac{2 \varepsilon^{1-2 \sigma}}{\varphi^{\prime \prime}(0)}\left(\frac{V_{\eta \eta}}{V}-\frac{\left(V_{\eta}\right)^{2}}{V^{2}}\right), \quad \frac{\partial u_{\mathrm{in}}}{\partial t}=-\frac{2 \varepsilon^{1-\mu-\sigma}}{\varphi^{\prime \prime}(0)}\left(\frac{V_{\eta \theta}}{V}-\frac{V_{\eta} V_{\theta}}{V^{2}}\right)
$$

in terms of the inner variables $\eta=\frac{x}{\varepsilon^{\sigma}}$ and $\theta=\frac{t}{\varepsilon^{\mu}}$, where the functional factors of the powers of $\varepsilon$ have a finite order in the domain $\Omega_{\varepsilon}^{\varepsilon^{\sigma}}$, since

$$
\left|x \varepsilon^{-\varkappa}\right|+|t|=\left|\eta \varepsilon^{\sigma-\varkappa}\right|+\left|\theta \varepsilon^{\mu}\right|=\varepsilon^{\mu}(|\eta|+|\theta|) .
$$

Then estimate (1.11) becomes

$$
\frac{\partial u_{\text {in }}}{\partial t}+\frac{\partial \varphi\left(u_{\text {in }}\right)}{\partial x}-\varepsilon \frac{\partial^{2} u_{\text {in }}}{\partial x^{2}}=O\left(\varepsilon^{3-4 \sigma}\right), \quad \varepsilon \rightarrow+0,
$$

and, taking into account that $1-\mu-\sigma=2-3 \sigma$ by (1.6), we find the exact order $\varepsilon^{2-3 \sigma}$ of derivatives entering into the denominator in formula (1.10). Thus, we obtain the necessary estimate in the right-hand side of (1.10), since $1-\sigma=\varkappa$ by (1.6).

Further, differentiating (1.8) with respect to $x$ and passing to the inner variable $\eta$, we derive the relation

$$
\frac{\partial^{l} u_{\text {in }}}{\partial x^{l}}=\frac{\varepsilon}{V^{l+1}} \sum_{m_{0}+\cdots+m_{l}=l+1} a_{m_{0} \ldots m_{l}} \frac{\partial^{m_{0}} V}{\partial x^{m_{0}}} \ldots \frac{\partial^{m_{l}} V}{\partial x^{m_{l}}}=\frac{\varepsilon^{1-(l+1) \sigma}}{V^{l+1}} \sum_{m_{0}+\cdots+m_{l}=l+1} a_{m_{0} \ldots m_{l}} \frac{\partial^{m_{0}} V}{\partial \eta^{m_{0}}} \ldots \frac{\partial^{m_{l}} V}{\partial \eta^{m_{l}}}
$$

with some constant coefficients $a_{m_{0} \ldots m_{l}}$. Using Laplace's method for the integral $V(x, t, \varepsilon)$, we write the equation

$$
4^{n}(S(\eta, \theta))^{2 n+1}-\theta S(\eta, \theta)+\frac{1}{2} \eta=0
$$


for critical points by equating the derivative of the argument of the exponent in (1.9) to zero and taking into account the change of variables (1.4). For $\theta=$ const, as $\eta \rightarrow-\infty$ we obviously obtain a unique critical point $s=S(\eta, \theta)>0$, while as $\eta \rightarrow+\infty$ we obtain a unique critical point $s=S(\eta, \theta)<0$. Then according to the standard formula [8, ch. II, sect. 2.4], we find the following asymptotics:

$$
\frac{\partial^{m_{j}} V}{\partial \eta^{m_{j}}} \sim|\eta|^{m_{j} /(2 n+1)}, \quad \eta \rightarrow \infty, \quad \frac{\partial^{l} u_{\text {in }}}{\partial x^{l}} \sim \varepsilon^{-l}|x|^{(l+1) /(2 n+1)}, \quad x \rightarrow \infty .
$$

Since in the domain $\Omega_{\varepsilon}$ the inner variables $\eta, \theta$ are finite and the function $V$ (together with its derivatives) does not depend on $\varepsilon$ in the leading order, by the definition (1.7) of the norm in the weighted Sobolev spaces $W_{p, q}^{m}(\mathbb{R})$, the above relations imply the asymptotic equivalence $\left\|u_{\text {in }}(\cdot, t, \varepsilon)\right\|_{W_{p, q}^{m}(\mathbb{R})} \sim \varepsilon^{-m}$ as $\varepsilon \rightarrow+0$ for any $p, q$, and $m$ satisfying the inequality $(m+1) p<$ $(2 n+1)(q-1)$. Theorem 2 is proved.

\section{Transition of a weak discontinuity into a strong one}

In papers $[9,10]$ the solution of problem $(0.1)-(0.2)$ is studied in the case when the initial function is smooth everywhere except for one point, at which it is continuous and has a jump discontinuity of the first derivative:

$$
q(x)=-\left(x+a x^{2}\right) \Theta(-x)\left(1+g_{1}(x)\right), \quad t_{0}=-1,
$$

where $a>0, g_{1} \in C^{\infty}(\mathbb{R}), g_{1}(x)=0$ in some neighborhood of zero, $\Theta$ denotes the Heaviside function. Then $\varphi^{\prime}(q(x))=-\left(x+b x^{2}\right) \Theta(-x)\left(1+g_{2}(x)\right)$, where $b=a-\varphi^{\prime \prime \prime}(0) / 2>0$, and such a weak discontinuity in the limit problem propagates a finite time along a characteristic, and then turns into a shock wave.

In a neighborhood of the singular point $(x=0, t=0)$ we introduce the stretched inner variables

$$
\xi=\varepsilon^{-2 / 3} x, \quad \tau=\varepsilon^{-1 / 3} t .
$$

The asymptotics of the solution has the form of the series

$$
u(x, t, \varepsilon)=\sum_{p=2}^{\infty} \varepsilon^{p / 6} \sum_{s=0}^{[p / 2]-1} w_{p, s}(\xi, \tau) \ln ^{s} \varepsilon, \quad \varepsilon \rightarrow+0 .
$$

The leading term of the expansion $\varepsilon^{1 / 3} w_{2,0}(\xi, \tau)$ is found using the Cole-Hopf transform (under the assumption that $\left.\varphi^{\prime \prime}(0)=1\right)$

$$
\begin{gathered}
w_{2,0}(\xi, \tau)=-\frac{2}{\Phi(\xi, \tau)} \frac{\partial \Phi(\xi, \tau)}{\partial \xi}, \\
\Phi(\xi, \tau)=\int_{0}^{+\infty} \exp \left(-\frac{4 b}{3} s^{3}+\tau s^{2}-\xi s\right) d s .
\end{gathered}
$$

The argument of the exponent corresponds to a versal deformation for the boundary singularity $B_{3}$, whose general form is $s^{3}+\lambda_{1} s^{2}+\lambda_{2} s+\lambda_{3}$, see [11], however, the factor $e^{\lambda_{3}}$ does not play a role in this case due to the form of the Cole-Hopf transform.

To describe the behavior of the functions $w_{p, s}(\xi, \tau)$ as $\xi^{2}+\tau^{2} \rightarrow \infty$, in the plane of independent variables the following overlapping domains are introduced:

$$
X^{0}=\left\{(\xi, \tau):|\xi|<|\tau|^{1-\gamma}, \tau<0\right\}, \quad X^{\mathrm{s}}=\left\{(\xi, \tau):\left|\xi-3 \tau^{2} / 16 b\right|<\tau^{2-\nu}, \tau>0\right\} .
$$




$$
\begin{gathered}
X^{-}=\left\{(\xi, \tau): \xi<0,-|\xi|^{2-\alpha}<\tau\right\} \cup\left\{(\xi, \tau): \tau>0,16 b \xi<3 \tau^{2}-\tau^{\alpha-1}\right\}, \\
X^{+}=\left\{(\xi, \tau): \xi>0,-\xi^{2-\alpha}<\tau \leqslant 0\right\} \cup\left\{(\xi, \tau): \tau>0,16 b \xi>3 \tau^{2}+\tau^{\alpha-1}\right\},
\end{gathered}
$$

where $0<\gamma<1 / 2$, parameters $\alpha$ and $\nu$ are chosen so that $0<\nu<\alpha<(1-2 \gamma) /(1-\gamma)<1$. Then the union $X^{+} \cup X^{0} \cup X^{-} \cup X^{\mathrm{s}}$ is a neighborhood of infinity in $\mathbb{R}^{2}$, see Figure 2 .

Note that the domain $X^{\mathrm{s}}$ contains the parabola $16 b \xi-3 \tau^{2}=0$, where the inner local maximum of the integrand in the right-hand side of (2.1) equals the boundary maximum. In each of these four domains the functions $w_{p, s}(\xi, \tau)$ have specific asymptotic behavior; corresponding series for the leading term $w_{2,0}(\xi, \tau)$ are given: in the domain $X^{0}$ by [10, Theorem 1], in the domain $X^{-}$by [10, Theorem 3], in the domain $X^{\mathrm{s}}$ by [10, Theorem 4], in the domain $X^{+}$by [10, Theorem 5].

Now, let us consider the following case, where the initial function is such that

$$
\varphi^{\prime}(q(x))=-\left(x+b x^{2 n}\right) \Theta(-x)\left(1+g_{2 n}(x)\right), \quad n \geqslant 1 .
$$

From the characteristic equation $x=y+(t+1) \varphi^{\prime}(q(y))$ with $y$ being a negative parameter and the relation $u \sim-y$ we have $x \approx-y t-b y^{2 n}$. Making a new change of variables (1.4), we obtain the equalities $\sigma=\varkappa+\mu=2 n \varkappa$. Taking into account relations (1.5), we find

$$
\sigma=\frac{2 n}{2 n+1}, \quad \mu=\frac{2 n-1}{2 n+1}, \quad \varkappa=\frac{1}{2 n+1} .
$$

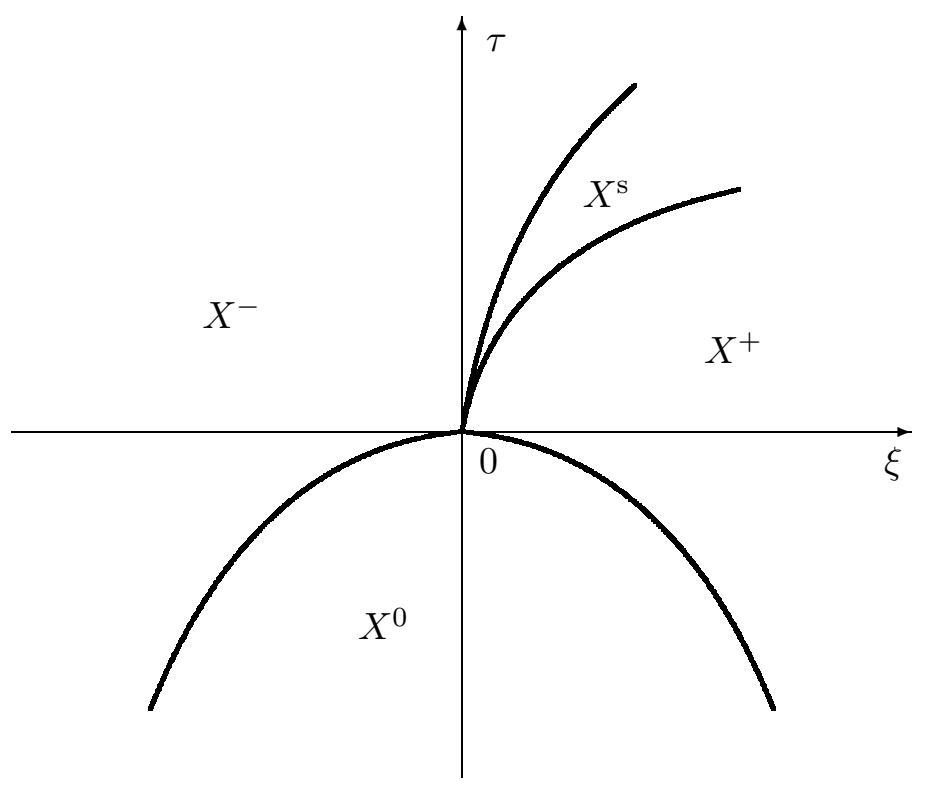

Fig. 2. Domains $X^{+}, X^{0}, X^{-}$, and $X^{\mathrm{s}}$.

By analogy with Theorem 2 we obtain the following result.

Theorem 3. In the domain $\Omega_{\varepsilon}^{\prime}=\left\{(x, t):\left|x \varepsilon^{-\varkappa}\right|+|t|<K \varepsilon^{\mu}, K>0\right\}$ for any natural $n \geqslant 2$ the function

$$
\begin{gathered}
u_{\mathrm{as}}(x, t, \varepsilon)=-2 \varepsilon[W(x, t, \varepsilon)]^{-1} \frac{\partial W(x, t, \varepsilon)}{\partial x}, \\
W(x, t, \varepsilon)=\int_{0}^{+\infty} \exp \left(-\frac{2^{2 n} b}{2 n+1} s^{2 n+1}+\frac{t s^{2}}{\varepsilon^{\mu}}-\frac{x s}{\varepsilon^{\sigma}}\right) d s,
\end{gathered}
$$


where numbers $\sigma, \mu$, and $\varkappa$ are defined by formulas (2.2), is an asymptotic solution of equation (0.1) in the following sense:

$$
\frac{\partial u_{\mathrm{as}} / \partial t+\partial \varphi\left(u_{\mathrm{as}}\right) / \partial x-\varepsilon \partial^{2} u_{\mathrm{as}} / \partial x^{2}}{\sup _{(x, t) \in \Omega_{\varepsilon}^{\prime}}\left\{\left|\partial u_{\mathrm{as}} / \partial t\right|+\left|\partial \varphi\left(u_{\mathrm{as}}\right) / \partial x\right|+\left|\varepsilon \partial^{2} u_{\mathrm{as}} / \partial x^{2}\right|\right\}}=O\left(\varepsilon^{\varkappa}\right), \quad \varepsilon \rightarrow+0 .
$$

For $(m+1) p<2 n(q-1)$ and any fixed $t, u_{\mathrm{as}}(\cdot, t, \varepsilon) \in W_{p, q}^{m}(\mathbb{R})$ and $\left\|u_{\mathrm{as}}(\cdot, t, \varepsilon)\right\|_{W_{p, q}^{m}(\mathbb{R})} \sim \varepsilon^{-m}$ as $\varepsilon \rightarrow+0$.

P r o o f. Formula (2.4) implies that the function $W(x, t, \varepsilon)$ satisfies the heat equation

$$
\frac{\partial W}{\partial t}=\varepsilon \frac{\partial^{2} W}{\partial x^{2}}
$$

it follows that function (2.3) is the exact solution of the Burgers equation:

$$
\frac{\partial u_{\mathrm{as}}}{\partial t}+u_{\mathrm{as}} \frac{\partial u_{\mathrm{as}}}{\partial x}=\varepsilon \frac{\partial^{2} u_{\mathrm{as}}}{\partial x^{2}}
$$

Then, using the relation $\varphi^{\prime}(u)=u+O\left(u^{2}\right), u \rightarrow 0$, we easily obtain the following estimate:

$$
\frac{\partial u_{\mathrm{as}}}{\partial t}+\frac{\partial \varphi\left(u_{\mathrm{as}}\right)}{\partial x}-\varepsilon \frac{\partial^{2} u_{\mathrm{as}}}{\partial x^{2}}=\varphi^{\prime}\left(u_{\mathrm{as}}\right) \frac{\partial u_{\mathrm{as}}}{\partial x}-u_{\mathrm{as}} \frac{\partial u_{\mathrm{as}}}{\partial x}=O\left(u_{\mathrm{as}}^{2} \frac{\partial u_{\mathrm{as}}}{\partial x}\right) .
$$

It is also elementary checked that

$$
u_{\mathrm{as}}^{2}=4 \varepsilon^{2-2 \sigma} \frac{\left(W_{\eta}\right)^{2}}{W^{2}}, \quad \frac{\partial u_{\mathrm{as}}}{\partial x}=-2 \varepsilon^{1-2 \sigma}\left(\frac{W_{\eta \eta}}{W}-\frac{\left(W_{\eta}\right)^{2}}{W^{2}}\right), \quad \frac{\partial u_{\mathrm{as}}}{\partial t}=-2 \varepsilon^{1-\mu-\sigma}\left(\frac{W_{\eta \theta}}{W}-\frac{W_{\eta} W_{\theta}}{W^{2}}\right)
$$

in terms of the inner variable $\eta=x / \varepsilon^{\sigma}$ and $\theta=t / \varepsilon^{\mu}$, where the functional factors of the powers of $\varepsilon$ have a finite order in the domain $\Omega_{\varepsilon}^{\prime}$, since $\left|x \varepsilon^{-\varkappa}\right|+|t|=\left|\eta \varepsilon^{\sigma-\varkappa}\right|+\left|\theta \varepsilon^{\mu}\right|=\varepsilon^{\mu}(|\eta|+|\theta|)$. Then, estimate (2.6) becomes

$$
\frac{\partial u_{\mathrm{as}}}{\partial t}+\frac{\partial \varphi\left(u_{\mathrm{as}}\right)}{\partial x}-\varepsilon \frac{\partial^{2} u_{\mathrm{as}}}{\partial x^{2}}=O\left(\varepsilon^{3-4 \sigma}\right), \quad \varepsilon \rightarrow+0,
$$

and, taking into account that $1-\mu-\sigma=2-3 \sigma$ by (2.2), we find the exact order $\varepsilon^{2-3 \sigma}$ of derivatives entering into the denominator in formula (2.5). Thus, we obtain the necessary estimate in the right-hand side of $(2.5)$, since $1-\sigma=\varkappa$ by $(2.2)$.

The estimate of the weighted norm $\left\|u_{\text {as }}(\cdot, t, \varepsilon)\right\|_{W_{p, q}^{m}(\mathbb{R})} \sim \varepsilon^{-m}$ as $\varepsilon \rightarrow+0$ is obtained almost exactly in the same way as in the proof of Theorem 2 .

In conclusion, it should be said that by using a priori estimates of solutions of the parabolic equation (0.1) one can prove that the asymptotic solutions defined by formulas (1.8), (1.9) and (2.3), (2.4) are close to some exact solutions; however, such a proof needs uniform formal asymptotic solutions of equation (0.1) in a whole strip $\left\{t_{0} \leqslant t \leqslant T, x \in \mathbb{R}\right\}$ containing a singular point to be constructed. 


\section{REFERENCES}

1. Arnold V. I. Singularities of Caustics and Wave Fronts. Ser. Math. Appl., Vol. 62. Netherlands: Springer, 1990. DOI: 10.1007/978-94-011-3330-2

2. Khesin B., Misiołek G. Shock waves for the Burgers equation and curvatures of diffeomorphism groups. Proc. Steklov Inst. Math., 2007. Vol. 259. P. 73-81. DOI: 10.1134/S0081543807040062

3. Il'in A. M. Matching of Asymptotic Expansions of Solutions of Boundary Value Problems. Transl. Math. Monogr., Vol. 102. Am. Math. Soc., 1992. 281 p.

4. Zakharov S. V. Singularities of $A$ and $B$ types in asymptotic analysis of solutions of a parabolic equation. Funct. Anal. Appl., 2015. Vol. 49, No. 4. P. 307-310. DOI: 10.1007/s10688-015-0120-1

5. Il'in A. M. The Cauchy problem for a quasilinear parabolic equation with a small parameter. Dokl. Akad. Nauk SSSR, 1985. Vol. 283, No. 3. P. 530-534.

6. Arnol'd V.I. Normal forms for functions near degenerate critical points, the Weyl groups of $A_{k}, D_{k}, E_{k}$ and Lagrangian singularities. Funct. Anal. Appl., 1972. Vol. 6, No. 4. P. 254-272. DOI: $10.1007 /$ BF01077644

7. Zakharov S. V. Asymptotic solution of the multidimensional Burgers equation near a singularity. Theoret. and Math. Phys., 2018. Vol. 196, No. 1. P. 976-982. DOI: 10.1134/S0040577918070048

8. Erdélyi A. Asymptotic Expansions. New York: Dover Publ., 1956. 108 p.

9. Il'in A. M., Zakharov S. V. From weak discontinuity to gradient catastrophe. Sb. Math., 2001. Vol. 192, No. 10. P. 1417-1433. DOI: 10.1070/SM2001v192n10ABEH000599

10. Zakharov S. V. Asymptotic solution of a Cauchy problem in a neighbourhood of a gradient catastrophe. Sb. Math., 2006. Vol. 197, No. 6. P. 835-851. DOI: 10.1070/SM2006v197n06ABEH003780

11. Arnol'd V.I. Critical points of functions on a manifold with boundary, the simple Lie groups $B_{k}$, $C_{k}$, and $F_{4}$ and singularities of evolutes. Russian Math. Surveys, 1978. Vol. 33, No. 5. P. 99-116. DOI: 10.1070/RM1978v033n05ABEH002515 